\title{
Intention to Breastfeed Among Female Students of Tertiary Institutions In Ekiti State, Southwest Nigeria.
}

\author{
Olatunde Olatunde Ogundare ( $\square$ ogundarezra@gmail.com ) \\ Ekiti State University https://orcid.org/0000-0003-0082-0001 \\ Adefunke Olarinre Babatola \\ Ekiti State University College of Medicine \\ Adefunke Christianah Omoyajowo \\ Bowen University, Iwo, Osun State \\ Adebukola Bidemi Ajite \\ Ekiti State University College of Medicine \\ Adewuyi Temidayo Adeniyi \\ Ekiti State University College of Medicine \\ Odunayo Adebukola Fatunla \\ Ekiti State University Teaching Hospital \\ Omolayo Adebukola Olubosede \\ University of Medical Sciences Teaching Hospital, Akure, Ondo State
}

Adekunle Bamidele Taiwo

Ekiti State University Teaching Hospital, Ado-Ekiti, Ekiti State

Adedayo Adewumi Oluyi

Ekiti State University Teaching Hospital, Ado-Ekiti, Ekiti State

Ayotunde Emmanuel Ajibola

Ekiti State University Teaching Hospital, Ekiti State

Akinwumi Kolawole Komolafe

Ekiti State University Teaching Hospital, Ado-Ekiti, Ekiti State

Alfred Airemionkhale

Ekiti State University Teaching Hospital, Ado-Ekiti, Ekiti State

Isaac Oludare Oluwayemi

Ekiti State University College of Medicine

Oladele Simeon Olatunya

Ekiti State University College of Medicine

\section{Research}

Keywords: Breastfeeding, Female Students, Attitude, Tertiary institutions, Intention, Nigeria

Posted Date: August 31st, 2020

DOI: https://doi.org/10.21203/rs.3.rs-62324/v1

License: @) (i) This work is licensed under a Creative Commons Attribution 4.0 International License. Read Full License 


\section{Abstract}

Introduction: Breastfeeding is a public health practice that is critical for the survival of infants and young children.

Despite the well documented advantages of breastfeeding to both the child and mother, it is surprising to note that the rate of breastfeeding especially exclusive breastfeeding is quite low in many countries. The possible reason may be because most of the efforts and activities directed at improving the breastfeeding rate and duration are targeted at pregnant women and post-partum period.

This study aimed at determining the knowledge, attitude, beliefs, and future intention to breastfeed among female undergraduates in tertiary institutions in Ekiti State, South West Nigeria.

Methods: The study was a prospective cross-sectional study conducted among female students of tertiary institutions in Ekiti State. By convenience sampling, female students were invited to participate in the study after giving verbal consent and those that were thirty years old and above, medical or nursing students were excluded. The questionnaire used for this study was self-administered. The proforma was designed to assess the students' knowledge about breastfeeding, attitude, beliefs, and future intention towards breastfeeding.

Data analysis was with SPSS version 25 using descriptive and inferential statistics. Results were summarized as percentages. Consensus index was calculated for the questions on the knowledge of the benefits of breastfeeding and the perception of breastfeeding.

Result: One thousand, three hundred and twelve (1312) students participated in the study. Only $58 \%$ of the respondents defined exclusive breastfeeding correctly. The participants displayed a particularly good knowledge of the importance of breast milk for the brain growth as $93 \%$ of the respondents agreed that breast milk is important for the development of the infant's brain. As much as $51 \%$ of the respondents agreed that breast milk is not needed after infancy. Only $34 \%$ of the respondents indicated their intention to breastfeed their future babies, $6.5 \%$ said no.

Conclusion: This study revealed that most of the future mothers have poor knowledge and beliefs about breastfeeding as well as negative attitude toward breastfeeding, hence there is need to step up awareness about breastfeeding and its benefits among this huge population of future mothers.

\section{Introduction:}

Breastfeeding is a public health practice that is critical for the survival of infants and young children. It has potential of preventing about 804,000 deaths per annum (13.8\% of all deaths) among children under two years of age in developing world [1]. Breastmilk contains essential nutrients, vitamins, and antibodies that are important for the optimal growth, development, and health of an infant. It also contains a diverse mixture of bioactive components that influence the immune system of an infant by facilitating development, tolerance, and an appropriate inflammatory response, in addition to providing necessary protection [2]. Breastfed children have at least six times greater chance of survival in the early months than non-breastfed children [3]. An exclusively breastfed child is 14 times less likely to die in the first six months than a nonbreastfed child [3].

Breastfeeding drastically reduces deaths from acute respiratory infection and diarrhea which are two major child killers. ${ }^{3}$ Breastfeeding also enhances optimal physical growth and cognitive development of children. Studies have documented other benefits of breastfeeding which include reducing the risk of asthma, food allergies and type II diabetes later in adult life $[4,5,6,7,8]$. Furthermore, breastfeeding impacts positively on the health of mothers; reduces the risk of postpartum hemorrhage (PPH), prevents breast cancer, cardiovascular diseases, rheumatoid arthritis, assists in child spacing through delay fertility (lactational amenorrhoea); It also promotes bonding between baby and mother [4-8].

Based on the numerous benefits that can be derived from breastfeeding, the World Health Organization [9] (WHO) recommends that women should breastfeed for a period of two (2) years and beyond, which includes initiating breastfeeding within an hour of delivery of the infant, breastfeeding exclusively for the first six months of life and continuing breastfeeding in addition to complementary feeding till 2 years. Also, organizations and governments around the world support and promote this age long practice to ensure that more women engage in the act.

Despite the well documented advantages of breastfeeding to both the child and mother, it is surprising to note that the rate of breastfeeding especially exclusive breastfeeding is quite low in many countries. In Nigeria where this study was conducted, only 29 percent of infants less than 6 months of age were exclusively breastfed based on the 2018 Nigeria Demographic and Health Survey (NDHS) data [10]. Even, globally, the exclusive breastfeeding rate is just about $43 \%$ [11]. The possible reason may be because most of the efforts and activities directed at improving the breastfeeding rate and duration are targeted at pregnant women and post-partum period [12, 13].

The present approach has not yielded the desired result despite years of its practice, hence there is a need for a paradigm shift and this may be to educate would-be mothers such as female undergraduates or the younger age groups about breastfeeding and its benefits knowing fully well that the knowledge acquired will influence their attitude towards breastfeeding and breastfeeding practices later in life. Breastfeeding practices

Page 2/23 
in various cross-cultural settings have been predicted using the theory of planned behaviour which states that intention to breastfeed precedes the breastfeeding behaviour manifested by an individual [14]. Intention itself has been documented to be influenced by attitude, knowledge, exposure, subjective norms and perceived behavioural control $[15,16]$.

The infant feeding practices and decisions are constantly being made within a woman's socio-cultural context and most of the opinions and decisions about breastfeeding are likely to have been made before conception [17]. Since the decisions and opinions about breastfeeding are formed well before conception, it may be more profitable to target the young ladies, trying to understand their knowledge base, beliefs, misconceptions and perceptions of breastfeeding which may serve as hindrances to the uptake and successful practice of breastfeeding in that huge population [18]. This population of future mothers have been studied extensively in developed countries, however most of the studies conducted in developing countries had focused on medical and nursing students who by the virtue of their professional studies should have some knowledge about breastfeeding $[19,20,21]$. There are very few studies on intention to breastfeed among female non-medical undergraduates in developing countries like Nigeria, hence the reason for this study.

It is also believed that the study will help in identifying gaps that still exist which are hindering progress at increasing the exclusive breastfeeding rate in Ekiti State and in Nigeria at large. It is believed that findings from this study will help those saddled with the responsibility of policy formulation and everyone involved in supporting and promoting breastfeeding to re-strategize and focus on this promising group so as to be able to improve on the current appalling breastfeeding rates as it is being recorded every year.

This study is therefore aimed at determining the knowledge, attitude, beliefs, and future intention to breastfeed among female undergraduates in tertiary institutions in Ekiti State, South West Nigeria.

\section{Materials And Methods}

The study was a prospective cross-sectional study conducted among female students of tertiary institutions in Ekiti State. Five tertiary institutions in Ekiti State were purposively selected; two were Federal Government owned institutions, two State owned institution and one private institution. The choice of these institutions was based on the believe that academic and social inequalities to exist among those attending tertiary institutions in Nigeria. By convenience sampling, female students were invited to participate in the study after giving verbal consent and those that were thirty years old and above, medical or nursing students were excluded. The questionnaire used for this study was self-administered. The enquiries were designed based on the review of literature; these included the students' knowledge about breastfeeding, attitude, beliefs, and future intention towards breastfeeding. The students indicated their level of agreement or disagreement regarding breastfeeding and its benefits to the infant and the mother on a modified five-point Likert's scale for agreement. Most of the questions were framed positively with 'strongly agree' reflecting a positive attitude towards breastfeeding. However, some questions were negatively framed in which 'strongly disagree' reflects positive attitude towards breastfeeding. The intention to breastfeed their future children was assessed as a closed-ended question. The first section of the questionnaire comprised of the sociodemographic of the participants, the second section assessed the knowledge of the participants about the different terminologies associated with infant feeding, the third section assessed the participants knowledge of the benefits of breastfeeding, the fourth section sought to determine the beliefs and perception of the participants about breastfeeding while the fifth section was to determine their intention to breastfeed their baby and the duration.

The study was conducted between June and October 2019.

\section{Data Analysis:}

Data analysis was with SPSS version 25 using descriptive and inferential statistics. Results were summarized as percentages. Consensus index was calculated for the questions on the knowledge of the benefits of breastfeeding and the perception of breastfeeding. Consensus index greater than $80 \%$ was considered as good consensus. The differences in knowledge, intention and other responses among the participants were compared among age groups, faculties and with their sources of information using Chi-square test. The p-value of $<0.05$ was considered as statistically significant.

\section{Ethical consideration}

Ethical approval was obtained from the Ethics and Research Committees of the Ekiti State University Teaching Hospital, Ado-Ekiti.

\section{Results}

One thousand, three hundred and twelve (1312) students participated in the study.

The mean age of the participants was 20.93 years $( \pm 3.33)$. About forty-eight percent of the respondents were aged between 20 and 24 years. Over $88 \%$ of the participants were single while less than $1 \%$ were divorced (Fig. 1). Twelve percent of the study participants already had a child. 
Expectedly $79.2 \%$ of the respondents were of Yoruba origin. Only $1.5 \%$ of the respondents were postgraduate students. Twenty seven percent of the respondents were from the Faculty of Social Sciences, $19.4 \%$ each from the faculties of Science and Management while less than $5 \%$ each were from the Faculties of Agriculture, Education and Engineering. Other demographic characteristics of the respondents are as shown in Table 1. 
Table 1

The Socio-demographic characteristics of the respondents

\begin{tabular}{|c|c|c|}
\hline Characteristics & Frequency $(\mathrm{N})$ & Percentage (\%) \\
\hline \multicolumn{3}{|l|}{ Age (in years) } \\
\hline $15-19$ & 429 & 32.7 \\
\hline $20-24$ & 626 & 47.7 \\
\hline $25-29$ & 169 & 12.9 \\
\hline No Response & 88 & 6.7 \\
\hline Total & 1312 & 100.0 \\
\hline Mean & $20.9( \pm 3.33)$ & \\
\hline \multicolumn{3}{|l|}{ Highest Qualification } \\
\hline SSCE & 298 & 22.7 \\
\hline National Diploma & 121 & 9.2 \\
\hline NCE & 41 & 3.1 \\
\hline Bachelor of Science & 145 & 11.1 \\
\hline No Response & 707 & 53.9 \\
\hline Total & 1312 & 100.0 \\
\hline \multicolumn{3}{|l|}{ Religion } \\
\hline Christianity & 1023 & 78.0 \\
\hline Islam & 261 & 19.9 \\
\hline Traditional & 22 & 1.7 \\
\hline No Response & 6 & 0.5 \\
\hline Total & 1312 & 100.0 \\
\hline \multicolumn{3}{|l|}{ Tribe } \\
\hline Yoruba & 1039 & 79.2 \\
\hline Ibo & 126 & 9.6 \\
\hline Hausa & 57 & 4.3 \\
\hline Ebira & 22 & 1.7 \\
\hline Tiv & 12 & .9 \\
\hline ljaw & 6 & .5 \\
\hline Idoma & 11 & .8 \\
\hline Urhobo & 5 & .4 \\
\hline No Response & 34 & 2.6 \\
\hline Total & 1312 & 100.0 \\
\hline \multicolumn{3}{|l|}{ Marital Status } \\
\hline Single & 1157 & 88.2 \\
\hline Married & 149 & 11.4 \\
\hline No Response & 6 & 0.4 \\
\hline Total & 1312 & 100.0 \\
\hline \multicolumn{3}{|l|}{ Do you have a child? } \\
\hline Yes & 157 & 12.0 \\
\hline
\end{tabular}

Page 5/23 


\begin{tabular}{|lll|}
\hline Characteristics & Frequency $(\mathrm{N})$ & Percentage (\%) \\
\hline No & 647 & 49.3 \\
\hline No Response & 508 & 38.7 \\
\hline Total & 1312 & 100.0 \\
\hline Faculty of Study & & \\
\hline Science & 255 & 19.4 \\
\hline Agriculture & 35 & 2.7 \\
\hline Arts & 201 & 15.3 \\
\hline Social Sciences & 361 & 27.5 \\
\hline Engineering & 42 & 3.2 \\
\hline Management & 255 & 19.4 \\
\hline Education & 56 & 4.3 \\
\hline No Response & 107 & 8.2 \\
\hline Total & 1312 & 100.0 \\
\hline
\end{tabular}

\section{Respondents' knowledge of the definition of different pattern of infant feeding}

Only fifty eight percent of the respondents defined exclusive breastfeeding correctly, $34 \%$ of the respondents erroneously defined water administration in addition to breastfeeding in the first 6 months of life as exclusive breastfeeding. Twenty-five percent of the respondents defined predominant breastfeeding appropriately while only $18 \%$ defined prolonged breastfeeding appropriately (Table 2 ).

Table 2

Knowledge of definition of different Infant Feeding Methods/ Patterns

\begin{tabular}{|lllll|}
\hline Feeding Pattem/ Responses & Correct Response (\%) & Incorrect Response (\%) & Don't Know (\%) & No Response (\%) \\
\hline Exclusive Breastfeeding & $772(58.8)$ & $401(30.6)$ & $128(9.8)$ & $11(0.8)$ \\
\hline Predominant Breastfeeding & $329(25.1)$ & $809(61.7)$ & $118(9.0)$ & $56(4.3)$ \\
\hline Complementary Feeding & $191(14.6)$ & $973(74.2)$ & $93(7.1)$ & $55(4.2)$ \\
\hline Prolonged Breastfeeding & $242(18.4)$ & $776(59.1)$ & $239(18.2)$ & $55(4.2)$ \\
\hline
\end{tabular}

\section{Respondents knowledge of breastfeeding and its benefits}

The participants displayed a particularly good knowledge of the importance of breast milk for the brain growth as $93 \%$ of the respondents agreed that breast milk is important for the development of the infant's brain. This is the only question with a good consensus index score above 80. Only $53 \%$ of the respondents agreed that breast milk alone is sufficient for infants below 6 months of age. Regarding the other benefits of breastfeeding to the infant, $58 \%$ of the respondents agreed that breastfeeding reduces the risk of obesity, Type II DM and allergies in the infant while for the benefits of breastfeeding to the mother, the respondents demonstrated average knowledge in this regard, as only $56 \%$ agreed that breastfeeding reduces the risk of some cancers in the woman while approximately $52 \%$ agreed that breastfeeding reduces the risk of depression in the mother. The respondents demonstrated poor understanding of the definition of exclusive breastfeeding as $56 \%$ of them agreed that infants need to be given water in addition to breast milk right from birth. The consensus index for most of the knowledge tested in the section fell below 80 (Table 3). 
Table 3

Assessment of knowledge of Breastmilk and Benefits of Breastfeeding

\begin{tabular}{|c|c|c|c|c|c|}
\hline Benefits of Breastfeeding & Agree* (\%) & $\begin{array}{l}\text { Neither Agree nor } \\
\text { Disagree (\%) }\end{array}$ & $\begin{array}{l}\text { Disagree+ } \\
(\%)\end{array}$ & $\begin{array}{l}\text { No } \\
\text { Response } \\
(\%)\end{array}$ & $\begin{array}{l}\text { Consensus } \\
\text { Index (\%) }\end{array}$ \\
\hline Breast milk is important for development of the brain & $1220(93.0)$ & $61(4.6)$ & 21(1.6) & $10(0.8)$ & 86.55 \\
\hline Breast milk is a Newborn's "first vaccine" & 1032(78.7) & 207(15.8) & $51(2.2)$ & 22(1.7) & 73.23 \\
\hline $\begin{array}{l}\text { Colostrum is very rich in antibodies, which help babies } \\
\text { fight infection }\end{array}$ & 1047(79.8) & $155(11.8)$ & $88(6.7)$ & 22(1.7) & 69.41 \\
\hline Breast milk only is adequate for the first 6 months of life & $700(53.3)$ & $269(20.5)$ & 278(21.2) & $65(5.0)$ & 48.91 \\
\hline $\begin{array}{l}\text { Breastfeeding reduces risks of Obesity, Type II diabetes } \\
\text { mellitus and allergies }\end{array}$ & $761(58.0)$ & $217(16.5)$ & 132(10.0) & $148(11.3)$ & 43.29 \\
\hline Encourages bonding between mother and her baby & $878(67.0)$ & 286(21.8) & 125(9.6) & $2(0.2)$ & 67.02 \\
\hline It prevents some cancers in women & $739(56.3)$ & $280(21.3)$ & 118(9.0) & $121(9.2)$ & 48.40 \\
\hline Reduces risk of maternal depression & $677(51.6)$ & 294(22.4) & 145(11.0) & $122(9.3)$ & 48.84 \\
\hline Improves baby's cognition & $824(62.8)$ & $156(11.9)$ & 175(13.3) & $115(8.8)$ & 44.29 \\
\hline $\begin{array}{l}\text { A baby needs to drink water apart from breast milk from } \\
\text { birth }\end{array}$ & $743(56.6)$ & 193(14.7) & 261(19.9) & $61(4.6)$ & 44.49 \\
\hline *- Combination of strongly agreed and Agreed & & & & & \\
\hline
\end{tabular}

+ - Combination of Disagreed and Strongly Disagreed

\section{Respondents' perception of breastfeeding and breast milk}

The respondents' perception of breastfeeding and breast milk was below average overall. Thirty-nine percent of the respondents believed that colostrum is dirty and should be discarded; only $34 \%$ of the participants disagreed. Thirty-seven percent of the respondents agreed that exclusive breastfeeding prevents infants from eating complementary food later in life. As much as $51 \%$ of the respondents agreed that breast milk is not needed after infancy. Only $35 \%$ of the study participants agreed that rooming in should be practiced from delivery. Thirty eight percent of the study participants agreed that the size of woman's breast determines the volume of breast milk she produces. Close to $47 \%$ of the participants agreed that breastfeeding is not fashionable, $52 \%$ agreed that breastfeeding will make the breast to sag. Concerning the use of pacifiers hindering breastfeeding, $32 \%$ of the respondents agreed that pacifiers will not interfere with breastfeeding practices (Table 4 ). 
Table 4

Perception of Breastfeeding and Breast milk

\begin{tabular}{|c|c|c|c|c|c|}
\hline Perception of Breastfeeding/ Responses & $\begin{array}{l}\text { Agree* } \\
(\%)\end{array}$ & $\begin{array}{l}\text { Neither Agree } \\
\text { nor Disagree } \\
(\%)\end{array}$ & $\begin{array}{l}\text { Disagree+ } \\
(\%)\end{array}$ & $\begin{array}{l}\text { No } \\
\text { Response } \\
(\%)\end{array}$ & $\begin{array}{l}\text { Consensus } \\
\text { Index (\%) }\end{array}$ \\
\hline $\begin{array}{l}\text { Colostrum (first milk produced) is dirty milk that should be } \\
\text { discarded }\end{array}$ & $514(39.2)$ & 209(15.9) & $442(33.7)$ & $147(10.4)$ & 40.75 \\
\hline $\begin{array}{l}\text { A mother should be allowed to rest for a greater part of the day } \\
\text { after delivery before commencing breastfeeding }\end{array}$ & $575(43.8)$ & 293(22.3) & $296(22.6)$ & $32(2.4)$ & 54.7 \\
\hline $\begin{array}{l}\text { A breastfeeding mother needs adequate hydration to sustain } \\
\text { lactation }\end{array}$ & $656(60.0)$ & 268(20.4) & $240(18.3)$ & $44(3.4)$ & 51.97 \\
\hline $\begin{array}{l}\text { Exclusive Breastfeeding prevents infants from eating adult food } \\
\text { later }\end{array}$ & $484(36.9)$ & 142(10.8) & 493(37.5) & $56(4.3)$ & 47.39 \\
\hline Rooming-in should be practiced from delivery & $465(35.4)$ & $277(21.1)$ & 232(17.7) & $190(4.5)$ & 48.26 \\
\hline Breast milk is not necessary beyond the first 12 months of life & $669(50.9)$ & 203(15.5) & $280(21.3)$ & $32(2.4)$ & 52.66 \\
\hline $\begin{array}{l}\text { The size of one's breast will determine the quantity of breast milk } \\
\text { produced }\end{array}$ & $498(38.0)$ & 206(15.7) & $434(33.0)$ & $131(10.0)$ & 54.79 \\
\hline Breastfeeding is not fashionable & $610(46.5)$ & 163(12.4) & $376(28.6)$ & $42(3.2)$ & 48.29 \\
\hline Use of pacifiers will not interfere with breastfeeding practices & $420(32.0)$ & $285(21.7)$ & 257(19.6) & $190(14.5)$ & 42.07 \\
\hline Breastfeeding in public is offensive & $440(33.5)$ & 202(15.4) & $470(35.9)$ & $64(4.9)$ & 49.13 \\
\hline Breastfeeding is stressful and takes time & $522(39.8)$ & 154(11.7) & $488(37.2)$ & $32(2.4)$ & 48.59 \\
\hline Breastfeeding makes the breast to sag & $677(51.6)$ & $164(12.5)$ & $351(26.8)$ & $27(2.1)$ & 50.61 \\
\hline Breastfeeding give the mother body odour & $338(25.7)$ & 179(13.6) & $649(49.5)$ & $42(3.2)$ & 54.44 \\
\hline
\end{tabular}

\section{+ - Combination of Disagreed and Strongly Disagreed}

\section{Respondents' sources of information about breastmilk and breastfeeding}

Thirteen percent of the respondents got their information about breastfeeding from the sayings of old women who are mostly mothers and the grandmothers in the family, about $10 \%$ from online/ media sites and literatures on breastfeeding, $4.4 \%$ from hospital or clinic, approximately $4 \%$ from friends while as much as $59 \%$ of the participants did not respond to the question (Fig. 1).

\section{Respondents' knowledge of exclusive breastfeeding and appropriate breastfeeding initiation time}

Respondents demonstrated poor knowledge as to the recommended time for commencement of breastfeeding after the delivery of a baby as only $4.0 \%$ of the respondents agreed that newborn babies should commence breastfeeding within one hour of delivery, about $11 \%$ agreed to $1-6$ hours after delivery, $7 \%$ agreed to more than 6 hours after delivery while $78 \%$ of the study participants did not know the appropriate time when a newborn baby should initiate breastfeeding after delivery

The study participants demonstrated poor understanding of exclusive breastfeeding as only about $14 \%$ agreed that water should be introduced to exclusively breastfed infants at the 6 th month of life, $8 \%$ of the respondents believed that water can be introduced right from birth while $41 \%$ do not know when water should be introduced to an infant on exclusive breastfeeding (Fig. 2).

About forty-nine percent of the respondents agreed that babies should be exclusively breastfed after delivery, $14 \%$ did not believe that babies should be exclusively breastfed while close to $1 \%$ are undecided, however $36 \%$ of participants gave no response.

\section{Respondents' intention to breastfeed}

When the participants were asked about the intention to breastfeed their future babies, thirty four percent of the respondents intend to breastfeed their future babies, $6.5 \%$ said no. As high as $58 \%$ of the study participants did not respond to this question (Fig. 3 ).

In terms of exclusive breastfeeding, $44 \%$ of the respondents intend to breastfeed their future babies exclusively, $14 \%$ do not intend to practice exclusive breastfeeding while as high as $42 \%$ did not respond to the question. 
Respondents who had their information from literatures demonstrated poor knowledge of the of the correct definition of exclusive breastfeeding and complementary feeding. These findings were statistically significant. $(p=0.000)$ Respondents' who had their information from friends demonstrated poor knowledge of the correct definition of prolonged breastfeeding. This finding was statistically significant $(p=0.000)$.

For the Faculty of study, respondents from the Faculty of Engineering demonstrated statistically significant poor knowledge of the definition of exclusive breastfeeding and prolonged breastfeeding $(p=0.000)$. Respondents' from the faculty of Engineering demonstrated statistically significant poor knowledge of the definition of predominant breastfeeding $(p=0.000)$. Respondents' from the faculty of Education demonstrated statistically significant poor knowledge of the correct definition of complementary feeding. $(p=0.000)$ This finding was statistically significant (Table 5). 
Table 5

Relationship between Respondents' knowledge of definition of infant feeding patterns and Sources of information and Faculty of study

\begin{tabular}{|c|c|c|c|c|c|c|c|c|c|c|}
\hline \multirow[t]{2}{*}{ DEFINITIONS } & \multicolumn{5}{|c|}{ Exclusive Breastfeeding } & \multicolumn{5}{|c|}{ Predominant Breastfeeding } \\
\hline & Correct & Incorrect & $\begin{array}{l}\text { I don't } \\
\text { know }\end{array}$ & Total (\%) & $\begin{array}{l}\text { P- } \\
\text { Value }\end{array}$ & Correct & Incorrect & $\begin{array}{l}\text { I don't } \\
\text { know }\end{array}$ & $\begin{array}{l}\text { Total } \\
(\%)\end{array}$ & $\begin{array}{l}\text { P- } \\
\text { Value }\end{array}$ \\
\hline \multirow{2}{*}{$\begin{array}{l}\text { Source of } \\
\text { Information (\%) }\end{array}$} & \multirow{2}{*}{$\begin{array}{l}120 \\
(68.2)\end{array}$} & $38(21.6)$ & \multirow{2}{*}{$\begin{array}{l}18 \\
(10.2)\end{array}$} & $176(33.1)$ & \multirow[t]{8}{*}{0.000} & \multirow{2}{*}{$\begin{array}{l}40 \\
(24.8)\end{array}$} & \multirow{2}{*}{$\begin{array}{l}111 \\
(68.9)\end{array}$} & $10(6.2)$ & \multirow{2}{*}{$\begin{array}{l}161 \\
(31.5)\end{array}$} & \multirow[t]{8}{*}{0.000} \\
\hline & & $28(22.0)$ & & $127(23.9)$ & & & & $1(0.8)$ & & \\
\hline $\begin{array}{l}\text { Saying of older } \\
\text { women }\end{array}$ & $\begin{array}{l}94 \\
(74.0)\end{array}$ & 62 (51.7) & \multirow{2}{*}{$\begin{array}{l}5(3.9) \\
16 \\
(13.3)\end{array}$} & $120(226)$ & & $\begin{array}{l}30 \\
(23.6)\end{array}$ & $\begin{array}{l}96 \\
(75.6)\end{array}$ & & $\begin{array}{l}127 \\
(24.9)\end{array}$ & \\
\hline Media/Online sites & $\begin{array}{l}42 \\
(35.0)\end{array}$ & $22(43.1)$ & & $51(9.6)$ & & \multirow[t]{2}{*}{$\begin{array}{l}28 \\
(24.6)\end{array}$} & \multirow[t]{2}{*}{$\begin{array}{l}70 \\
(61.4)\end{array}$} & $\begin{array}{l}(14.0) \\
3(5.9)\end{array}$ & \multirow[t]{2}{*}{$\begin{array}{l}114 \\
(22.3)\end{array}$} & \\
\hline \multirow{2}{*}{$\begin{array}{l}\text { Literature on } \\
\text { breastfeeding }\end{array}$} & \multirow{2}{*}{$\begin{array}{l}26 \\
(51.0)\end{array}$} & $20(34.5)$ & $3(5.9)$ & 58 (10.9) & & & & & & \\
\hline & & $170(32.0)$ & $0(0.0)$ & $532(100.0)$ & & $\begin{array}{l}24 \\
(47.1)\end{array}$ & $\begin{array}{l}24 \\
(47.1)\end{array}$ & $\begin{array}{l}14 \\
(24.1)\end{array}$ & $\begin{array}{l}51 \\
(10.0)\end{array}$ & \\
\hline Hospitals/Clinics & \multicolumn{2}{|l|}{$\begin{array}{l}38 \\
(65.5)\end{array}$} & \multicolumn{2}{|l|}{$42(7.9)$} & & $\begin{array}{l}14 \\
(24.1)\end{array}$ & $\begin{array}{l}30 \\
(51.7)\end{array}$ & $44(8.6)$ & $\begin{array}{l}58 \\
(11.4)\end{array}$ & \\
\hline Total (\%) & $\begin{array}{l}320 \\
(60.2)\end{array}$ & & & & & $\begin{array}{l}136 \\
(26.6)\end{array}$ & $\begin{array}{l}331 \\
(64.8)\end{array}$ & & $\begin{array}{l}478 \\
(100.0)\end{array}$ & \\
\hline Student's Faculty & 173 & $56(22.0)$ & 26 & $255(21.4)$ & 0.000 & 50 & 160 & 30 & 240 & 0.000 \\
\hline$(10)$ & (01.0) & $65(32.3)$ & & $201(16.8)$ & & & & & & \\
\hline Science & $\begin{array}{l}116 \\
(57.7)\end{array}$ & $105(30.0)$ & $0(0.0)$ & $350(2.9)$ & & $\begin{array}{l}43 \\
(21.5)\end{array}$ & $\begin{array}{l}141 \\
(70.5)\end{array}$ & $16(8.0)$ & $\begin{array}{l}200 \\
(17.4)\end{array}$ & \\
\hline & 211 & $16(38.1)$ & $\begin{array}{l}20 \\
(10.0)\end{array}$ & $42(3.5)$ & & & 237 & $23(6.9)$ & 333 & \\
\hline Social Sciences & $(60.3)$ & 100 (39.2) 5 & $34(9.7)$ & $255(21.4)$ & & & & $\begin{array}{l}16 \\
(50.0)\end{array}$ & & \\
\hline Engineering & $\begin{array}{l}10 \\
(23.8)\end{array}$ & $(14.3)$ & & $35(2.9)$ & & $0(0.0)$ & 16 & $15(5.9)$ & $32(2.8)$ & \\
\hline Management & & $15(26.8)$ & $(38.1)$ & $56(4.7)$ & & 109 & & & 255 & \\
\hline Agriculture & $(54.9)$ & $82(16.0)$ & $15(5.9)$ & $1194(100.0)$ & & & (51.4) & $0(14.0)$ & & \\
\hline Education & 30 & & $2(3.6)$ & & & (14.3) & 25 & $2(4)-3$ & J & \\
\hline Total (\%) & & & 113 & & & 11 & & (9.3) & 3 & \\
\hline & $\begin{array}{l}39 \\
(69.6)\end{array}$ & & $(9.5)$ & & & & $\begin{array}{l}43 \\
(76.8)\end{array}$ & & $\begin{array}{l}1151 \\
(100.0)\end{array}$ & \\
\hline & $\begin{array}{l}719 \\
(60.2)\end{array}$ & & & & & (25.3) & $\begin{array}{l}753 \\
(65.4)\end{array}$ & & & \\
\hline & Compler & entary Feeding & & & & Prolong & Breastfee & ing & & \\
\hline & Correct & Incorrect & $\begin{array}{l}\text { I don't } \\
\text { know }\end{array}$ & Total (\%) & $\begin{array}{l}\text { P- } \\
\text { Value }\end{array}$ & Correct & Incorrect & $\begin{array}{l}\text { I don't } \\
\text { know }\end{array}$ & $\begin{array}{l}\text { Total } \\
(\%)\end{array}$ & $\begin{array}{l}\text { P- } \\
\text { Value }\end{array}$ \\
\hline Source of & 28 & $116(74.4)$ & $12(7.7)$ & $156(30.5)$ & 0.000 & 36 & 96 & 24 & 156 & 0.000 \\
\hline о) & & $110(86.6)$ & $1(0.8)$ & $127(24.8)$ & & & & & & \\
\hline $\begin{array}{l}\text { Saying of older } \\
\text { women }\end{array}$ & $\begin{array}{l}16 \\
(12.6)\end{array}$ & $96(80.0)$ & & $120(23.4)$ & & $\begin{array}{l}32 \\
(25.2)\end{array}$ & $\begin{array}{l}90 \\
(70.9)\end{array}$ & $5(3.9)$ & $\begin{array}{l}127 \\
(24.8)\end{array}$ & \\
\hline & & & (13.3) & & & & & 26 & & \\
\hline Media/Online sites & $8(6.7)$ & $36(70.6)$ & $3(59)$ & $51(10.0)$ & & 22 & 72 & & 120 & \\
\hline Literature on & 12 & $38(65.5)$ & & $58(11.3)$ & & & & 23 & & \\
\hline & $(20.0)$ & 396 (77.3) & $4(0.9)$ & $512(100.0)$ & & $U(U$. & $(54.9)$ & & $(10.0)$ & \\
\hline & & & & & & $\begin{array}{l}18 \\
(31.0)\end{array}$ & $\begin{array}{l}22 \\
(37.9)\end{array}$ & & & \\
\hline & & & & & & $\begin{array}{l}108 \\
(21.1)\end{array}$ & 308 & & & \\
\hline & $\begin{array}{l}16 \\
(27.6)\end{array}$ & & $36(7.0)$ & & & & $(60.2)$ & $\begin{array}{l}18 \\
(31.0)\end{array}$ & 58 & \\
\hline Hospitals/Clinics & 80 & & & & & & & 96 & $(11.3)$ & \\
\hline Total (\%) & (15.6) & & & & & & & (18.8) & $\begin{array}{l}512 \\
(100.0)\end{array}$ & \\
\hline
\end{tabular}




\begin{tabular}{|c|c|c|c|c|c|c|c|c|c|c|}
\hline \multirow[t]{2}{*}{ DEFINITIONS } & \multicolumn{5}{|c|}{ Exclusive Breastfeeding } & \multicolumn{5}{|c|}{ Predominant Breastfeeding } \\
\hline & Correct & Incorrect & $\begin{array}{l}\text { I don't } \\
\text { know }\end{array}$ & Total (\%) & $\begin{array}{l}\text { P- } \\
\text { Value }\end{array}$ & Correct & Incorrect & $\begin{array}{l}\text { I don't } \\
\text { know }\end{array}$ & $\begin{array}{l}\text { Total } \\
(\%)\end{array}$ & $\begin{array}{l}\text { P- } \\
\text { Value }\end{array}$ \\
\hline \multirow{2}{*}{$\begin{array}{l}\text { Student's Faculty } \\
(\%)\end{array}$} & \multirow{2}{*}{$\begin{array}{l}61 \\
(26.1)\end{array}$} & $158(67.5)$ & $15(6.4)$ & $234(20.3)$ & \multirow[t]{11}{*}{0.000} & \multirow{2}{*}{$\begin{array}{l}86 \\
(36.8)\end{array}$} & \multirow{2}{*}{$\begin{array}{l}123 \\
(52.6)\end{array}$} & \multirow{2}{*}{$\begin{array}{l}25 \\
(10.7)\end{array}$} & \multirow{2}{*}{$\begin{array}{l}234 \\
(20.3)\end{array}$} & \multirow[t]{11}{*}{0.000} \\
\hline & & $149(74.1)$ & $20(0.0)$ & $201(17.4)$ & & & & & & \\
\hline Science & $\begin{array}{l}32 \\
(15.9)\end{array}$ & $299(86.9)$ & 22 & 344 (29.9) & & $\begin{array}{l}18 \\
(9.0)\end{array}$ & $\begin{array}{l}157 \\
(78.1)\end{array}$ & $\begin{array}{l}26 \\
(12.9)\end{array}$ & $\begin{array}{l}201 \\
(17.4)\end{array}$ & \\
\hline \multirow{2}{*}{$\begin{array}{l}\text { Arts } \\
\text { Social Sciences }\end{array}$} & \multirow{2}{*}{$\begin{array}{l}23 \\
(6.7)\end{array}$} & $16(50.0)$ & $(10.0)$ & $32(2.8)$ & & \multirow{2}{*}{$\begin{array}{l}55 \\
(16.0)\end{array}$} & \multirow{2}{*}{$\begin{array}{l}226 \\
(65.7)\end{array}$} & \multirow{2}{*}{$\begin{array}{l}63 \\
(18.3)\end{array}$} & \multirow{2}{*}{$\begin{array}{l}344 \\
(29.9)\end{array}$} & \\
\hline & & 185 (72.5) 25 & $6(18 . \varepsilon$ & $255(22.1)$ & & & & & & \\
\hline \multirow{3}{*}{ Management } & $\begin{array}{l}10 \\
(31.3)\end{array}$ & $(83.3)$ & $25(9.8)$ & $30(2.6)$ & & $0(0.0)$ & $\begin{array}{l}16 \\
(500)\end{array}$ & $\begin{array}{l}16 \\
(500)\end{array}$ & $32(2.8)$ & \\
\hline & \multirow{2}{*}{$\begin{array}{l}45 \\
(17.6)\end{array}$} & \multirow{5}{*}{$82(16.0)$} & $0(0.0)$ & $56(4.9)$ & & \multirow{2}{*}{$\begin{array}{l}46 \\
(18.0)\end{array}$} & & & \multirow{2}{*}{$\begin{array}{l}255 \\
(22.1)\end{array}$} & \\
\hline & & & $2(3.6)$ & $00(+) 190$. & & & $(52.5)$ & $(29.4)$ & & \\
\hline \multirow{3}{*}{$\begin{array}{l}\text { Education } \\
\text { Total (\%) }\end{array}$} & $\begin{array}{l}5 \\
(16.7)\end{array}$ & & $90(7.8)$ & $1102(100.0)$ & & $\begin{array}{l}5 \\
(16.7)\end{array}$ & $\begin{array}{l}20 \\
(66.7)\end{array}$ & $5(16.7)$ & $30(2.6)$ & \\
\hline & $2(3.6)$ & & & & & $\begin{array}{l}9 \\
(16.1)\end{array}$ & 39 & $8(14.3)$ & \multirow{2}{*}{$\begin{array}{l}56(4.9) \\
1152 \\
(100.0)\end{array}$} & \\
\hline & \multicolumn{2}{|l|}{$\begin{array}{l}178 \\
(15.5)\end{array}$} & & & & $\begin{array}{l}219 \\
(19.0)\end{array}$ & $\begin{array}{l}(09.0) \\
715 \\
(62.1)\end{array}$ & $(18.9)$ & & \\
\hline
\end{tabular}

\section{Relationship between knowledge of the benefits of breastfeeding and faculty of study}

A significantly higher proportion of the respondents agreed that breastfeeding improves the baby's cognition. Respondents from the Faculty of Agriculture demonstrated good knowledge of some of the benefits of breastfeeding. This finding was statistically significantly $(p=0.000)$. About $3 / 5$ th of the respondents from all the faculties agreed that breastfeeding prevents some cancers in women and reduces the risk of obesity, type II diabetes mellitus (DM) and allergies in children, this finding was statistically significant $(p=0.000)$. Most of the participants from the faculties of Education, Social science and Arts agreed that colostrum is dirty and should be discarded, this finding was significant statistically ( $p=0.000$.) Most respondents from the faculties of Agriculture and Arts agreed that the size of a woman's breast determines the volume of milk produced, this was also statistically significant $(p=0.000)$. Most respondents from the faculties of Science, Agriculture and Engineering agreed that breastfeeding is not fashionable and will make the breast to sag. This finding was significant statistically $(p=0.000)($ Table 6$)$. 
Table 6

Relationship between Respondents' Faculty of Study and knowledge of the benefits of Breastfeeding

\begin{tabular}{|c|c|c|c|c|c|c|c|c|c|c|}
\hline \multirow[t]{2}{*}{ RESPONSES } & \multicolumn{7}{|c|}{ Student's Faculty (\%) } & \multirow[t]{2}{*}{ Total } & \multirow{2}{*}{$\chi^{2}$} & \multirow{2}{*}{$\begin{array}{l}\mathrm{P} \text { - } \\
\text { value }\end{array}$} \\
\hline & Science & Agriculture & Arts & $\begin{array}{l}\text { Social } \\
\text { Sciences }\end{array}$ & Engineering & Management & Education & & & \\
\hline \multirow{3}{*}{$\begin{array}{l}\text { Breastfeeding } \\
\text { improves } \\
\text { baby's } \\
\text { cognition }\end{array}$} & $189(74.1)$ & $35(100.0)$ & 106 & 244 & $10(23.8)$ & $147(57.6)$ & $28(50.9)$ & \multirow{2}{*}{$\begin{array}{l}759 \\
(65.0)\end{array}$} & \multirow[t]{6}{*}{137.8} & \multirow[t]{6}{*}{0.000} \\
\hline & $25(9.8)$ & $0(0.0)$ & (דט) & & $10(23.8)$ & $50(19.6)$ & $17(30.9)$ & & & \\
\hline & $26(10.2)$ & $0(0.0)$ & $\begin{array}{l}32 \\
(16.3)\end{array}$ & $24(7.3)$ & $6(14.3)$ & $26(10.2)$ & $6(10.9)$ & $\begin{array}{l}158 \\
(13.5)\end{array}$ & & \\
\hline \multirow{3}{*}{$\begin{array}{l}\text { Agree } \\
\text { Disagree } \\
\text { Neutral } \\
\text { Don't Know } \\
\text { Total }\end{array}$} & \multirow{3}{*}{$\begin{array}{l}15(5.9) \\
255 \\
(100.0)\end{array}$} & \multirow{2}{*}{$\begin{array}{l}0(0.0) \\
35(100.0)\end{array}$} & $\begin{array}{l}38 \\
(19.4)\end{array}$ & $\begin{array}{l}41(12.4) \\
21(6.4)\end{array}$ & $16(38.1)$ & $32(12.5)$ & $4(7.3)$ & $\begin{array}{l}143 \\
(12.2)\end{array}$ & & \\
\hline & & & $\begin{array}{l}20 \\
(10.2)\end{array}$ & $\begin{array}{l}330 \\
(100.0)\end{array}$ & $42(100.0)$ & $255(100.0)$ & $\begin{array}{l}55 \\
(100.0)\end{array}$ & $\begin{array}{l}(1<.<) \\
108 \\
(9.2)\end{array}$ & & \\
\hline & & & $\begin{array}{l}196 \\
(100.0)\end{array}$ & & & & & $\begin{array}{l}1168 \\
(100.0)\end{array}$ & & \\
\hline \multirow{3}{*}{$\begin{array}{l}\text { Breastfeeding } \\
\text { prevents } \\
\text { cancers in } \\
\text { women. }\end{array}$} & 1133(52.2) & $30(85.7)$ & 122 & 241 & $21(50.0)$ & $101(39.6)$ & $38(69.1)$ & \multirow{2}{*}{$\begin{array}{l}686 \\
(59.3)\end{array}$} & \multirow[t]{6}{*}{136.4} & \multirow[t]{6}{*}{0.000} \\
\hline & $30(11.8)$ & $0(0.0)$ & & & $0(0.0)$ & $26(10.2)$ & $2(3.6)$ & & & \\
\hline & $56(22.0)$ & $0(0.0)$ & $\begin{array}{l}16 \\
(8.2)\end{array}$ & 33 (10.4) & 15 (35.7) & $90(35.3)$ & $14(25.5)$ & $\begin{array}{l}107 \\
(9.3)\end{array}$ & & \\
\hline & $36(14.1)$ & 5 (14.3) & 38 & & $6(14.3)$ & $38(14.9)$ & $1(1.8)$ & 251 & & \\
\hline Neutral & \multirow[t]{2}{*}{$\begin{array}{l}255 \\
(100.0)\end{array}$} & \multirow[t]{2}{*}{$35(100.0)$} & $\begin{array}{l}20 \\
(10.2)\end{array}$ & $\begin{array}{l}318 \\
(100.0)\end{array}$ & $42(100.0)$ & $255(100.0)$ & $\begin{array}{l}55 \\
(100.0)\end{array}$ & $\begin{array}{l}112 \\
(9.7)\end{array}$ & & \\
\hline Total & & & $\begin{array}{l}196 \\
(100.0)\end{array}$ & & & & & $\begin{array}{l}1156 \\
(100.0)\end{array}$ & & \\
\hline \multirow{4}{*}{$\begin{array}{l}\text { Breastfeeding } \\
\text { reduces the } \\
\text { risk of } \\
\text { obesity, Type } \\
\text { II DM and } \\
\text { allergies }\end{array}$} & $145(58.0)$ & $20(57.1)$ & 100 & 200 & $26(61.9)$ & $166(65.1)$ & $35(63.6)$ & \multirow{2}{*}{$\begin{array}{l}692 \\
(60.1)\end{array}$} & \multirow[t]{7}{*}{67.6} & \multirow[t]{7}{*}{0.000} \\
\hline & $20(8.0)$ & $0(0.0)$ & & & $10(23.8)$ & $26(10.2)$ & $2(3.6)$ & & & \\
\hline & $55(22.0)$ & $5(14.3)$ & $\begin{array}{l}26 \\
(13.3)\end{array}$ & & $0(0.0)$ & 45 (17.6) & $16(29.1)$ & $\begin{array}{l}117 \\
(10.2)\end{array}$ & & \\
\hline & $30(12.0)$ & $10(28.6)$ & 28 & & $6(14.3)$ & $18(7.1)$ & $2(3.6)$ & 197 & & \\
\hline Disagreed & $\begin{array}{l}250 \\
(100.0)\end{array}$ & 35 (100.0) & $\begin{array}{l}42 \\
(21.4)\end{array}$ & $\begin{array}{l}318 \\
(100.0)\end{array}$ & $42(100.0)$ & $255(100.0)$ & $\begin{array}{l}55 \\
(100.0)\end{array}$ & $\begin{array}{l}145 \\
(12.6)\end{array}$ & & \\
\hline Don't Know & & & $\begin{array}{l}196 \\
(100.0)\end{array}$ & & & & & $\begin{array}{l}1151 \\
(100.0)\end{array}$ & & \\
\hline Total & & & & & & & & & & \\
\hline Colostrum is & $92(38.5)$ & $15(42.9)$ & 100 & 169 & $10(27.0)$ & $63(26.4)$ & $35(66.0)$ & 484 & 245.6 & 0.000 \\
\hline should be & $126(52.7)$ & $10(28.6)$ & & & $16(43.2)$ & 76 (31.8) & $6(11.3)$ & & & \\
\hline discarded & $5(2.1)$ & $5(14.3)$ & $\begin{array}{l}45 \\
(24.9)\end{array}$ & $\begin{array}{l}111 \\
(33.1)\end{array}$ & $11(29.7)$ & $100(41.8)$ & $12(22.6)$ & $\begin{array}{l}390 \\
(34.9)\end{array}$ & & \\
\hline & $16(6.7)$ & $5(14.3)$ & 16 & $43(12.8)$ & $0(0.0)$ & $0(0.0)$ & $0(0.0)$ & 192 & & \\
\hline & 239 & $35(100.0)$ & & $12(3.6)$ & $37(100.0)$ & $239(100.0)$ & 53 & & & \\
\hline Iveutial & & & $(11.0)$ & 335 & & & & $\begin{array}{l}53 \\
(4.7)\end{array}$ & & \\
\hline Don't Know & & & 181 & (100.0) & & & & 111 & & \\
\hline Total & & & (100.0) & & & & & (100.0) & & \\
\hline
\end{tabular}




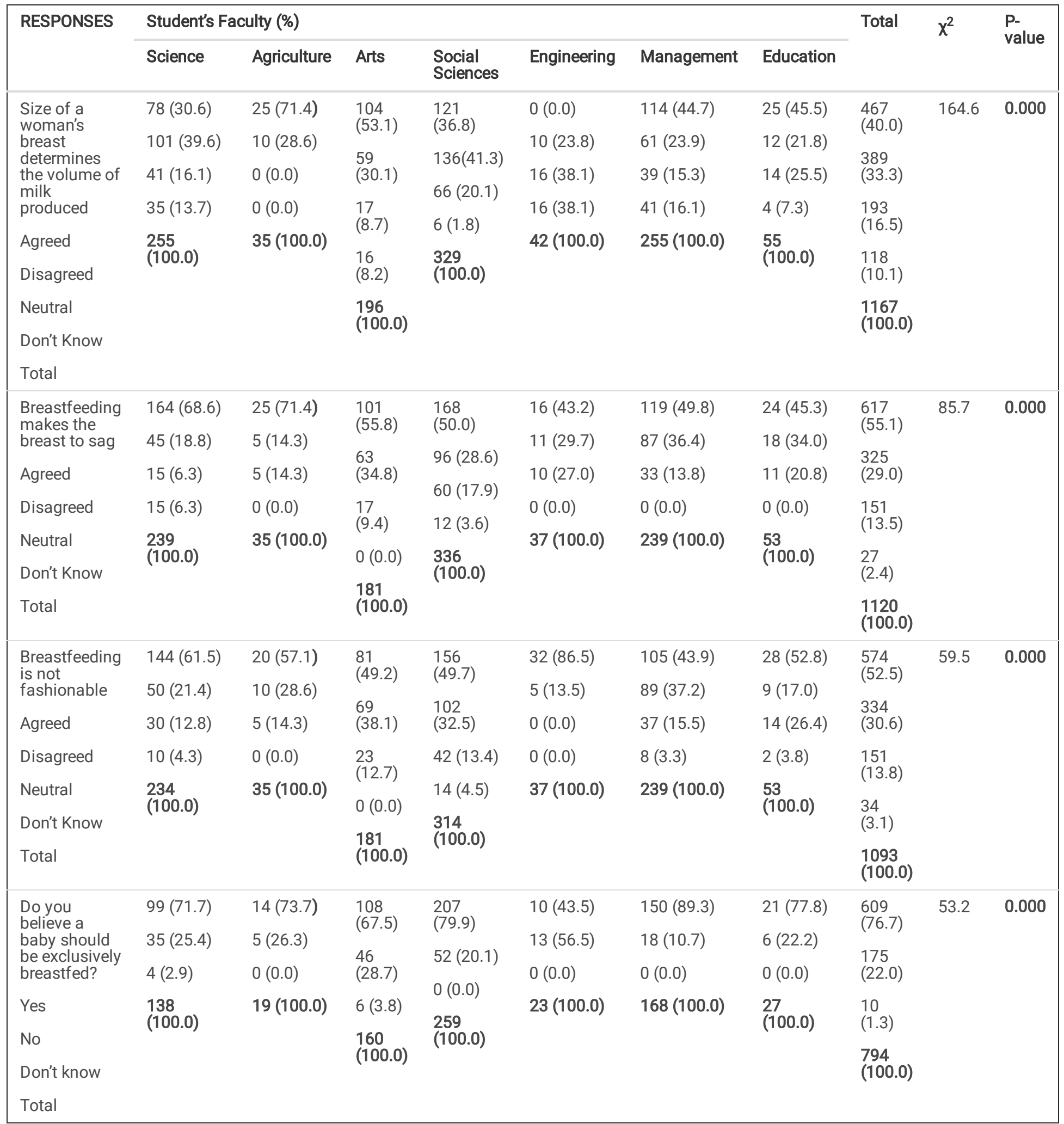

\section{Relationship between knowledge of the benefits of Breastfeeding and Sources of information}

Most of the participants despite the different sources of information agreed that breastfeeding improves the cognition of the child and reduces the risk of obesity, type II DM and allergies. Most respondents whose sources of information were from sayings of old women, Hospital/Clinics and Media/Online sites agreed that breastfeeding improves the cognition of the child and this finding was statistically significant $(p=0.000)$. Most respondents whose source of information was from friends were undecided with regards to breastfeeding preventing some cancers in women and reducing the risk of obesity, type II DM and allergies. A higher proportion of respondents who got their information from the hospital or clinics disagreed that colostrum should be discarded, and this was also significant statistically $(p=0.000)$. Most respondents' who agreed that the size of a woman's breast determine the volume of milk produced got their information from media/online sites. $p=0.000$. A higher 
proportion who disagreed that breastfeeding is not fashionable had their information from literature and sayings of old women. $(p=0.000)$ (Table 7). 
Table 7

Relationship between Respondents' Knowledge of the benefits of Breastfeeding and Sources of information

\begin{tabular}{|c|c|c|c|c|c|c|c|c|}
\hline \multirow[t]{2}{*}{ RESPONSES } & \multicolumn{8}{|c|}{ Sources of Information (Percentage) } \\
\hline & $\begin{array}{l}\text { Sayings of Old } \\
\text { women }\end{array}$ & $\begin{array}{l}\text { Media/ } \\
\text { Online sites }\end{array}$ & $\begin{array}{l}\text { Lit on } \\
\mathrm{B} / \mathrm{F}^{*}\end{array}$ & Friends & $\begin{array}{l}\text { Hospital } \\
\text { /Clinics }\end{array}$ & Total & $x^{2}$ & $\begin{array}{l}\mathrm{P} \text { - } \\
\text { value }\end{array}$ \\
\hline \multirow{2}{*}{$\begin{array}{l}\text { Breastfeeding improves baby's cognition } \\
\text { Agree }\end{array}$} & $134(81.7)$ & $86(67.7)$ & \multirow{2}{*}{$\begin{array}{l}72 \\
(59.0)\end{array}$} & $\begin{array}{l}18 \\
(35.3)\end{array}$ & \multirow{2}{*}{$\begin{array}{l}42 \\
(72.4)\end{array}$} & \multirow{2}{*}{$\begin{array}{l}352 \\
(67.4)\end{array}$} & \multirow[t]{7}{*}{90.0} & \multirow[t]{7}{*}{0.000} \\
\hline & $18(11.0)$ & $22(17.3)$ & & \multirow{2}{*}{$4(7.8)$} & & & & \\
\hline Disagree & $8(4.9)$ & $18(14.2)$ & $\begin{array}{l}26 \\
(21.3)\end{array}$ & & $4(6.9)$ & $\begin{array}{l}74 \\
(14.2)\end{array}$ & & \\
\hline Neutral & $4(2.4)$ & $1(0.8)$ & 14 & $\begin{array}{l}18 \\
(35.3)\end{array}$ & $4(6.9)$ & 62 & & \\
\hline Don't Know & $164(100.0)$ & $127(100.0)$ & (1 & \multirow{2}{*}{$\begin{array}{l}11 \\
(21.6)\end{array}$} & 0 & $(1, .3)$ & & \\
\hline \multirow[t]{2}{*}{ Total } & & & $(8.2)$ & & $\begin{array}{l}58 \\
(100.0)\end{array}$ & $\begin{array}{l}34 \\
(6.5)\end{array}$ & & \\
\hline & & & $\begin{array}{l}122 \\
(100.0)\end{array}$ & $\begin{array}{l}51 \\
(100.0)\end{array}$ & & $\begin{array}{l}522 \\
(100.0)\end{array}$ & & \\
\hline Breastfeeding prevents cancers in women. & $110(67.1)$ & $82(64.6)$ & $\begin{array}{l}70 \\
(61.4)\end{array}$ & $\begin{array}{l}10 \\
(19.6)\end{array}$ & \multirow{2}{*}{$\begin{array}{l}28 \\
(48.3)\end{array}$} & \multirow{2}{*}{$\begin{array}{l}300 \\
(58.4)\end{array}$} & \multirow[t]{6}{*}{154.8} & \multirow[t]{6}{*}{0.000} \\
\hline Agree & $10(6.1)$ & $26(20.5)$ & & \multirow{2}{*}{$0(0.0)$} & & & & \\
\hline Disagree & $12(7.3)$ & $15(11.8)$ & \multirow{2}{*}{$\begin{array}{l}8(7.0) \\
32 \\
(28.1)\end{array}$} & & $\begin{array}{l}10 \\
(17.2)\end{array}$ & $\begin{array}{l}54 \\
(10.5)\end{array}$ & & \\
\hline Neutral & $32(19.5)$ & $4(3.1)$ & & $(72.5)$ & $\begin{array}{l}12 \\
(20.7)\end{array}$ & $\begin{array}{l}108 \\
(21.0)\end{array}$ & & \\
\hline Don't Know & $164(100.0)$ & $127(100.0)$ & $4(3.5)$ & $4(7.8)$ & & & & \\
\hline Total & & & $\begin{array}{l}114 \\
(100.0)\end{array}$ & $\begin{array}{l}51 \\
(100.0)\end{array}$ & $\begin{array}{l}58 \\
(100.0)\end{array}$ & $\begin{array}{l}(10.1) \\
514 \\
(100.0)\end{array}$ & & \\
\hline Breastfeeding reduces the risk of obesity, & $106(64.6)$ & $84(66.1)$ & 56 & 16 & 50 & 312 & 129.9 & 0.000 \\
\hline & $10(6.1)$ & $16(12.6)$ & & & & & & \\
\hline Agreed & $30(18.3)$ & $7(5.5)$ & $\begin{array}{l}22 \\
(18.3)\end{array}$ & $0(0.0)$ & $4(6.9)$ & $\begin{array}{l}52 \\
(10.1)\end{array}$ & & \\
\hline Disagreed & $18(11.0)$ & $20(15.7)$ & 16 & $\begin{array}{l}29 \\
(64.4)\end{array}$ & & 86 & & \\
\hline Neutral & $164(100.0)$ & $127(100.0)$ & (13.3) & $0(0.0)$ & $0(0.0)$ & $(16.7)$ & & \\
\hline Don't Know & & & $\begin{array}{l}26 \\
(21.7)\end{array}$ & & $\begin{array}{l}58 \\
(100.0)\end{array}$ & $\begin{array}{l}64 \\
(12.5)\end{array}$ & & \\
\hline rotal & & & $\begin{array}{l}120 \\
(100.0)\end{array}$ & & & $\begin{array}{l}514 \\
(100.0)\end{array}$ & & \\
\hline Colostrum is dirty and should be discarded & $76(43.2)$ & $74(58.3)$ & 60 & $4(8.5)$ & 12 & 226 & 149.9 & 0.000 \\
\hline Agreed & $76(43.2)$ & $40(31.5)$ & & 12 & & & & \\
\hline Disagreed & $14(8.0)$ & $13(10.2)$ & $(27.6)$ & & $\begin{array}{l}32 \\
(55.6)\end{array}$ & $\begin{array}{l}192 \\
(36.6)\end{array}$ & & \\
\hline Neutral & $10(5.7)$ & $0(0.0)$ & 12 & $(66.0)$ & 14 & 84 & & \\
\hline Don't Know & $176(100.0)$ & $127(100.0)$ & & $0(0.0)$ & & $(16.0)$ & & \\
\hline Total & & & $\begin{array}{l}12 \\
(10.3)\end{array}$ & 47 & $0(0.0)$ & $\begin{array}{l}22 \\
(4.2)\end{array}$ & & \\
\hline & & & $\begin{array}{l}116 \\
(100.0)\end{array}$ & & $(100.0)$ & $\begin{array}{l}524 \\
(100.0)\end{array}$ & & \\
\hline
\end{tabular}




\begin{tabular}{|c|c|c|c|c|c|c|c|c|}
\hline RESPONSES & Sources of In & nation (Percen & ge) & & & & & \\
\hline Size of a woman's breast determines the & $68(40.0)$ & $74(58.3)$ & 56 & $4(7.8)$ & 12 & 214 & 119.0 & 0.000 \\
\hline Aared & $74(43.5)$ & $44(34.6)$ & & 14 & & & & \\
\hline Agreed & $16(9.4)$ & $8(6.3)$ & $\begin{array}{l}28 \\
(23.3)\end{array}$ & (27.5) & $\begin{array}{l}28 \\
(48.3)\end{array}$ & $\begin{array}{l}188 \\
(35.7)\end{array}$ & & \\
\hline Disagreed & $12(7.1)$ & $1(0.8)$ & 16 & $\begin{array}{l}25 \\
(51.0)\end{array}$ & 14 & 80 & & \\
\hline INeutral & $170(100.0)$ & $127(100.0)$ & $(13.3)$ & $7(13.7)$ & & $(15.2)$ & & \\
\hline & & & $(16.7)$ & $\begin{array}{l}51 \\
(100.0)\end{array}$ & $4(6.9)$ & $\begin{array}{l}44 \\
(8.4)\end{array}$ & & \\
\hline Don't Know & & & & & & & & \\
\hline Total & & & $\begin{array}{l}120 \\
(100.0)\end{array}$ & & $\begin{array}{l}58 \\
(100.0)\end{array}$ & $\begin{array}{l}526 \\
(100.0)\end{array}$ & & \\
\hline Breastfeeding makes the breast to sag & $126(71.6)$ & $84(66.1)$ & $\begin{array}{l}54 \\
(45.8)\end{array}$ & $\begin{array}{l}10 \\
(213)\end{array}$ & $\begin{array}{l}26 \\
(44.8)\end{array}$ & $\begin{array}{l}300 \\
(57.0)\end{array}$ & 104.4 & 0.000 \\
\hline Agreed & $38(21.6)$ & $22(17.3)$ & & 20 (62 0) & & & & \\
\hline Disagreed & $12(6.8)$ & $21(16.5)$ & $(33.9)$ & $7(149)$ & $(41.4)$ & $(29.3)$ & & \\
\hline Neutral & $0(0.0$ & $0(0.0)$ & 12 & $0(0 \cap)$ & $8(13.8)$ & 60 & & \\
\hline Don't Know & $176(100.0)$ & $127(100.0)$ & & & $0(0.0)$ & & & \\
\hline Total & & & $\begin{array}{l}12 \\
(10.2)\end{array}$ & $\begin{array}{l}47 \\
(100.0)\end{array}$ & 58 & $\begin{array}{l}12 \\
(2.3)\end{array}$ & & \\
\hline & & & $\begin{array}{l}118 \\
(100.0)\end{array}$ & & & $\begin{array}{l}526 \\
(100.0)\end{array}$ & & \\
\hline Breastfeeding is not fashionable & $80(47.1)$ & $84(66.1)$ & 54 & 24 & 38 & 280 & 52.5 & 0.000 \\
\hline Agreed & $60(35.3)$ & $26(20.5)$ & & & & & & \\
\hline Disagreed & $26(15.3)$ & $9(7.1)$ & $\begin{array}{l}38 \\
(35.8)\end{array}$ & $6(12.8)$ & $\begin{array}{l}20 \\
(34.5)\end{array}$ & $\begin{array}{l}150 \\
(29.5)\end{array}$ & & \\
\hline Neutral & $4(2.4)$ & $8(6.3)$ & 14 & $(27.7)$ & $0(0.0)$ & 62 & & \\
\hline Don't Know & $170(100.0)$ & $127(100.0)$ & 0 & $4(8.5)$ & $0(0.0)$ & & & \\
\hline Total & & & $0(0.0)$ & 47 & 58 & $\begin{array}{l}16 \\
(3.1)\end{array}$ & & \\
\hline & & & $(100.0)$ & $(100.0)$ & $(100.0)$ & $\begin{array}{l}508 \\
(100.0)\end{array}$ & & \\
\hline
\end{tabular}

\section{Relationship between intention to Breastfeed and Sources of information}

Most of the respondents intend to breastfeed their babies later in life irrespective of their source of information, all the respondents who got their information from hospitals or clinics intend to breastfeed their babies in the future $(p<0.001)($ Table 8$)$. 
Table 8

Relationship between Respondents' intention to breastfeed their future baby and Sources of information

\begin{tabular}{|c|c|c|c|c|c|c|c|c|}
\hline \multirow[t]{2}{*}{ RESPONSES } & \multicolumn{8}{|c|}{ Sources of Information (Percentage) } \\
\hline & $\begin{array}{l}\text { Sayings of Old } \\
\text { women }\end{array}$ & $\begin{array}{l}\text { Media/ Online } \\
\text { sites }\end{array}$ & $\begin{array}{l}\text { Lit on } \\
\mathrm{B} / \mathrm{F}^{*}\end{array}$ & Friends & $\begin{array}{l}\text { Hospital } \\
\text { /Clinics }\end{array}$ & Total & $x^{2}$ & $\begin{array}{l}\text { P- } \\
\text { value }\end{array}$ \\
\hline $\begin{array}{l}\text { Do you intend to breastfeed your } \\
\text { baby? }\end{array}$ & $146(83.0)$ & $90(76.9)$ & $92(78.0)$ & $\begin{array}{l}41 \\
(80.4)\end{array}$ & $\begin{array}{l}58 \\
(100.0)\end{array}$ & $\begin{array}{l}427 \\
(82.1)\end{array}$ & \multirow[t]{5}{*}{37.2} & \multirow[t]{6}{*}{0.000} \\
\hline Yes & $26(14.8)$ & $27(23.1)$ & $26(22.0)$ & $6(118)$ & $0(00)$ & $85(163)$ & & \\
\hline & $4(2.3)$ & $0(0.0)$ & $0(0.0)$ & & & & & \\
\hline No & \multirow{3}{*}{$176(100.0)$} & \multirow[t]{3}{*}{$117(100.0)$} & \multirow{3}{*}{$\begin{array}{l}118 \\
(100.0)\end{array}$} & $4(7.8)$ & $0(0.0)$ & $8(1.5)$ & & \\
\hline Undecided & & & & $\begin{array}{l}51 \\
(100.0)\end{array}$ & $\begin{array}{l}58 \\
(100.0)\end{array}$ & $\begin{array}{l}520 \\
(100.0)\end{array}$ & & \\
\hline Total & & & & & & & & \\
\hline $\begin{array}{l}\text { Will you exclusively breastfeed } \\
\text { your baby? }\end{array}$ & $120(80.0)$ & $81(65.9)$ & $74(74.0)$ & $\begin{array}{l}45 \\
(88.2)\end{array}$ & $\begin{array}{l}46 \\
(85.2)\end{array}$ & $\begin{array}{l}366 \\
(76.6)\end{array}$ & \multirow[t]{4}{*}{15.3} & \multirow[t]{4}{*}{0.004} \\
\hline Yes & \multirow{3}{*}{$150(100.0)$} & \multirow{3}{*}{$123(100.0)$} & \multirow{3}{*}{$\begin{array}{l}100 \\
(100.0)\end{array}$} & \multirow{3}{*}{$\begin{array}{l}6(11.8) \\
51 \\
(100.0)\end{array}$} & \multirow{3}{*}{$\begin{array}{l}8(14.8) \\
54 \\
(100.0)\end{array}$} & \multirow{2}{*}{$\begin{array}{l}112 \\
(23.4)\end{array}$} & & \\
\hline No & & & & & & & & \\
\hline Total & & & & & & $\begin{array}{l}4 / 8 \\
(100.0)\end{array}$ & & \\
\hline
\end{tabular}

A higher percentage of respondents aged between 15 and 19 years do not intend to breastfeed their babies. This finding was also statistically significant $p<0.000$ (Fig. 4).

\section{Discussion}

This study was designed to assess breastfeeding knowledge, beliefs, and perceived behavior and their impact on breastfeeding intention among female tertiary students in Ekiti State, Southwest Nigeria.

The study revealed that just a little above average of the respondents (58.8\%) had understanding of what exclusive breastfeeding (EBF) means, this is quite less than the $69 \%$ recorded by Shehu and Shehu in their study among clinical medical students in Jos [19].This is not unexpected as the participants in this study are not studying medical related courses as it has been documented that students in health-related majors have significantly higher levels of breastfeeding knowledge [22]. The finding may also be a true reflection of what obtains in the larger society. The study participants demonstrated poor understanding of the infant feeding practices. This is evident by the fact that only $25 \%$ of them had correct understanding of what predominant breastfeeding means, $14.6 \%$ for complementary feeding and $18.4 \%$ for prolonged breastfeeding. This result pattern shows that there is need to educate the future mothers on the different infant feeding practices and this could allow them to make a better decision later in life. As much as $34 \%$ of the respondents erroneously chose water administration in addition to breastfeeding in the first 6 months of life as exclusive breastfeeding and are likely to give their infants water prior to 6 months of life. This result shows that there is a knowledge gap regarding what exclusive breastfeeding means. Previous studies however did not evaluate these terminologies among their respondents.

The study showed that the study respondents had good understanding that breastmilk is important for brain development as that was the only response with consensus index above 80 which was considered an indication of good knowledge of the benefits of breastfeeding. Even though, the consensus index for other indices of the knowledge of the benefits of breastfeeding were higher than 50 , almost all the indices were below the score of 80 and this shows that gaps exist in their knowledge of breastfeeding and its benefits for both the child and mother and this may have effect on their perception of breastfeeding. This is not surprising as breastfeeding is not taught in elementary or secondary schools in Nigeria thus leaving these future mothers to acquire knowledge about breastfeeding and breast milk on their own or from unverified sources. Among the women of reproductive age groups, reliable information about breastfeeding are often times first heard during pregnancy during antenatal care provided that the lady registers for antenatal care and by which time many of them could have been wrongly informed and wrong opinions about breastfeeding already formed which might be difficult to change. The findings of this study are not different from that of Shehu and Shehu in Jos [19] among clinical medical students, Padmanabhan et al [23] among female college students in India and Anjun et al [24] among medical students in Pakistan.

Major knowledge gaps identified among these students included misconceptions that breast milk alone is inadequate for an infant in the first 6 months of life, water can be given to infants within the first 6 months of life, size of a woman's breast determines the volume of milk produced, exclusive breastfeeding can hinder infants from taking complementary feeds and that breastfeeding makes the breast to sag amongst others.

Page $17 / 23$ 
Interestingly, some of these misconceptions were also documented by previous studies; Hamade et al [25] documented similar finding among female undergraduate students in the middle East - Syria and Lebanon. Osman et al also documented similar findings among Lebanese mothers and concluded that many of these misconceptions were derived from the local culture [26]. The perception by about $50 \%$ of the study respondents that breastfeeding makes the breast to sag was also reported in the study by Nabulusi et al [27] among mothers in Lebanon; this was also reported by Hamade et al [25] in the study among female undergraduate university students in Lebanon and Syria. The major implication of this wrong perception is that these future mothers will likely discontinue breastfeeding earlier than the recommended time.

The opinion held by about 34\% of the participants that breastfeeding in the public is offensive is similarly high like the findings of Hamade et al [25] in which more than $50 \%$ of their study respondents had negative behavioural perception towards breastfeeding in the public and were against breastfeeding in public places or even in the presence of neighbors or friends in the home setting. This negative attitude towards breastfeeding in the public which probably is due to societal disapproval will not encourage breastfeeding especially exclusive breastfeeding. If the infant of such a mother cries for breast milk in a place the mother considers not secluded enough, she would probably give breastmilk substitute which would not only avoid "embarrassing" exposure of her breast but may also be erroneously considered a display of "class". These negative perceptions, coupled with the lack of public facilities to breastfeed comfortably, have ominous implications for breastfeeding and this may make it difficult to improve the exclusive breastfeeding rate in some developing countries, especially Nigeria. This concern has been raised by authors of previous studies [28, 29, 30,31], although studies investigating this issue in Nigeria are scanty.

Another knowledge gap identified by this study is that the study participants do not know how soon after delivery a newborn baby should commence breastfeeding. Only $4 \%$ of the respondents knew that newborn babies should be breastfed within one hour of delivery. This major knowledge gap puts the newborn at risk of hypoglycaemia and being offered pre-lacteal feeds, which increases the risk of sepsis, hence morbidity and mortality in such babies. This probably may be due to poor nutrition education among Nigerian students. There is need to integrate breastfeeding education into the nutrition curriculum right from the elementary school level to deepen the knowledge of the future parents on breastfeeding and associated benefits.

The source of information for most of the participants who responded to this question was mainly sayings of old women who are likely to be relatives such as mothers and grandmothers. Only $4.4 \%$ of the respondents got their information from the hospital or clinic, however $59 \%$ of the study participants did not respond to this question.

Most of the respondents showed good understanding of only exclusive breastfeeding irrespective of their source of information but majority have poor understanding of predominant breastfeeding, complementary feeding, and prolonged breastfeeding. Their knowledge of infant feeding practices was also not influenced by the faculty of study. These observations show that information has a role to play with understanding, hence the need to ensure that correct information about breastfeeding is passed across to the future mothers. The Faculty of study did not have effect on the participants knowledge of infant feeding practices, this might reflect the defect in the education system and the basic knowledge acquired by the participants during their elementary school days.

Intention is an important antecedent of behavior [25], and surprisingly, the intention to breastfeed by participants in this study is abysmally poor at $34 \%$ when compared with reports from the study by Shehu and Shehu in Jos [19] who reported intention to breastfeed of about $66 \%$ among clinical medical students. The study by Padmanabhan et al [23] in India showed as high $98.4 \%$ of the participants responding affirmatively, the study by Hamade et al [25] among Syrian and Lebanese female undergraduate university students reported $76.4 \%$ and $88.9 \%$ respondents respectively confirming their intention to breastfeed their babies. A major concern is the fact that $58.8 \%$ of our study participants did not respond to this simple question suggesting that they could be swayed in any direction depending on who counsels them. This study also showed that a higher percentage of the respondents aged 15 to 19 years do not intend to breastfeed their babies. This attitude is probably pointing to the fact that the culture of breastfeeding babies may be declining faster than expected and this portends a major problem for the future generation. This calls for urgent action to increase awareness about the breastfeeding and its benefits among the future mothers. Hamade et al [32] have previously documented that intention to breastfeed is one of the significant predictors of exclusive breastfeeding among Lebanese mothers. However, intention appears to be modulated by several other factors, including knowledge, attitude, and behavioral perceptions [16, 33, 34].Less than $50 \%$ of the respondents believe in exclusive breastfeeding and only $44 \%$ signified their intention to practice exclusive breastfeeding. This finding also portends grave danger to the practice of exclusive breastfeeding in the nearest future except urgent measure are taken to correct these negative attitudes. It has been shown further that improving parental knowledge, attitude, and behavioral perceptions toward breastfeeding can significantly improve not only the intention to breastfeed but also the initiation and duration of breastfeeding [7, 37, 36, 37].

This study probably highlights some of the reasons why it has been difficult for Nigeria to increase her exclusive breastfeeding rate and may depict what the future holds for breastfeeding in Nigeria. This study shows that the knowledge of most of these future mothers about breastfeeding and its benefits are not good enough and this may make it difficult for some of them to make the right/ best choice when the time comes for them to breastfeed their children. Since it has been shown that mothers with good knowledge about breastfeeding will likely have positive attitude towards breastfeeding and are also likely to be prepared to breastfeed their future children [22, 38], hence there is need to improve the knowledge of these future mothers. 
There are country-specific differences in the predictors of breastfeeding intention, this highlights the need to tailor breastfeeding intervention strategies to the local context and emphasizes the need for country-specific promotional tools to be used by breastfeeding advocates [25]. Even though culture specific interventions focusing on increasing knowledge and the promotion of the benefits of breastfeeding are needed to demystify some of the identified misconceptions about breastfeeding, there is also a need for implement and improve on the existing breastfeeding-friendly policies and legislation to encourage and support breastfeeding [25].

Also, the idea of waiting till antenatal period to educate would-be mothers about breastfeeding and its benefits is obviously not the best option, because some women do not attend antenatal care clinics at the appropriate health facilities as most of them prefer spiritual homes, traditional birth attendants (TBAs) and some women do not even attend antenatal care clinics which may render the current practice ineffective. Thus, there is a need for paradigm shift by starting the education of the future mothers about breastfeeding and its associated benefits early in life.

Measures targeted at young women during the early school years to increase awareness not only of the benefits of breastfeeding but also of the challenges encountered by breastfeeding women can be adopted [25]. Interventions may also include school curricula revision to ensure that breastfeeding is presented as the norm in texts, other resources, and during classroom discussions in elementary and secondary schools, in addition to encouraging universities to review and update their undergraduate and postgraduate training programs to reflect this [39]. School has been classified as the most important source of information by some authors and they have also been campaigning for the implementation of breastfeeding education in the school curriculum [40]. It has been shown that providing children with appropriate information on breastfeeding at school will probably make them better adults with greater capacity to choose the best nourishment for their children [41]. This may be one of the approaches that our nation has to take since this study has shown a fair knowledge and the perception of breastfeeding among female tertiary students and a below average intention to breastfeed and exclusively breastfeed their babies. Also, a reasonably high proportion of them were undecided whether to breastfeed their babies, these ones can be convinced to breastfeed their babies if given the right counsel.

Other intervention strategies may include the use of the media as a breastfeeding awareness vehicle, as it has been shown that attitudes, social norms, and cultural opinions about infant-feeding are partially shaped by the media including books, magazines, television, videos, and the internet [42]. By mitigating the societal taboos and promoting breastfeeding as a societal norm, the media may play an important role in addressing the issue of breastfeeding in public. More so, the media was the second highest source of information among participants in this study.

Nevertheless, the study was a cross sectional design study and this may affect its ability to predict the actual behaviour of the respondents, besides, being a self-administered questionnaire study, some questions were unanswered however this is not significant enough to invalidate the findings of the study. Also, the participants of the study are students of tertiary institutions who are believed to be educated and from urban areas, hence the findings may not be directly generalizable to all population groups.

The study focused on the female gender, however, it has been previously shown that male partners may exert an influence on infant-feeding decisions [43], hence it may be worth exploring the knowledge, beliefs and perception of young men about breastfeeding as well.

\section{Conclusion}

This study has shown that most of the future mothers have poor knowledge and beliefs about breastfeeding as well as negative attitude toward breastfeeding in the future. It has also become obvious that there is need to incorporate breastfeeding education into the current education curriculum at all levels with plausible effects on the present exclusive breastfeeding rate. Even though, breastfeeding a newborn baby is the norm in our society, the percentage of exclusively breastfed babies and children still taking breast milk after the first year of life are lower than expected. This study has shown that there are many misconceptions about breastfeeding and breast milk that require. Reviewing the curriculum to incorporate breastfeeding education at all levels may be one of the ways to do this, likewise, involvement of the mass media may also assist in achieving the desired goal. Also, improvement and implementation of existing legislation and policies targeted at increasing breastfeeding rates in the country need to be worked at to secure the future of the upcoming generation of babies.

\section{Abbreviations}

$\mathrm{PPH}$

Postpartum Haemorrhage

WHO

World Health Organization

NDHS

Nigeria Demographic and Health Survey

Type II DM

Type II Diabetes Mellitus

EBF 
Exclusive Breastfeeding

TBA

Traditional Birth Attendant

\section{Declarations}

ETHICS APPROVAL: Ethical approval was obtained from the Ethics and Research Committees of the Ekiti State University Teaching Hospital, Ado-Ekiti. The approval number is EKSUTH/A67/2019/05/0011.

AVAILABILITY OF DATA AND MATERIALS: The datasets used and/or analyzed during the current study are available from the corresponding author on reasonable request.

\section{COMPETING INTERESTS}

The authors declare that they have no competing interest.

\section{FUNDING:}

The authors declare that no funding was received for the study.

\section{AUTHORS' CONTRIBUTIONS:}

The study was conceived and designed by OEO together with BAO, OAC and FOA. OAA and OAC assisted with data collection. OAC, FOA and OEO analyzed the data. OEO wrote the initial draft. BAO, AAB, AAT, OAC, FOA, OOA, TAB, KAK, AA, OIO, OOS critically reviewed the manuscript. All authors read and approved the final draft of the manuscript.

\section{ACKNOWLEDGEMENTS}

The authors appreciate all the students who agreed to participate in the study as well as assistants who helped in the administration of the study proforma.

\section{References}

1. Victora C, Bahl R, Barros A, Franca GVA, Horton S, Krasevec J, Murch S, Sankar MJ, Walker N, Rollins NC. "Breastfeeding in the 21st Century: Epidemiology. Mechanisms Lifelong Effect" The Lancet. 2016;387(10017):475-90.

2. Field CJ. The immunological components of human milk and their effect on immune development in infants. J Nutr. 2005;135:1-4.

3. Black RE, Allen LH, Bhutta ZA, Caulfield LE, de Onis M, Ezzati M, Mathers C, Rivera J. Maternal and Child Undernutrition Group. Maternal and child undernutrition: global and regional exposures and health consequences. Lancet. 2008;371:243-60.

4. Jones G, Steketee R, Black R, Bhutta Z, Morris S. The Bellagio Child Survival Study Group. How many child deaths can we prevent this year? Lancet. 2003;362:65-71.

5. Chantry C, Howard C, Auinger P. Full breastfeeding duration and associated decrease in respiratory tract infection in US children. Pediatr. 2006;117:425-32.

6. Stuebe A. The Risks of Not Breastfeeding for Mothers and Infants. Rev Obstet Gynecol. 2009;2:222-31.

7. Dennis CL. Breastfeeding initiation and duration: a 1990-2000 literature review. J Obstet Gynecol Neonatal Nurs. 2002;31:12-32.

8. American Academy of Pediatrics Section on Breastfeeding. "Breastfeeding the use of human milk" Pediatrics. 2012;129(3):e827-41. 10.1542/peds.2011-3552. doi.

9. World Health Organization. (2001). The World Health Organization's infant feeding recommendation. http://www.who.int/nutrition/topics/infantfeeding_recommendation/en/index.html.

10. Nigeria Demographics and Health Survey (NDHS) 2018.

11. United Nations Children's Fund. Global breastfeed scorecard: Find out more about breastfeeding progress [Internet]. UNICEF. [cited 2020 Apr 12]. Available from: https://www.unicef.org/nutrition/index_100585.html.

12. Pavičić Bošnjak A, Grgurić J. Maternal characteristics influencing the duration of breastfeeding in a breastfeeding support group. Paediatr Croat. 2007;51:89-95.

13. Shi Y, Wang D, Yuan Y, Jiang Y, Zeng Q, Chang C. The effect of prenatal education curriculum on mother's prenatal examination utilization, delivery mode and recovery status: a cross-sectional survey in China. Environ Health Prev Med. 2015;20:397-403.

https://doi.org/10.1007/s12199-015-0480-4.

Page 20/23 
14. Dodgson JE, Henly SJ, Duckett L, Tarrant M. Theory of planned behavior-based models for breastfeeding duration among Hong Kong mothers. Nurs Res. 2003;52:148-58.

15. Kavanagh KF, Lou Z, Nicklas JC, Habibi MF, Murphy LT. Breastfeeding knowledge, attitudes, prior exposure, and intent among undergraduate students. J Hum Lact. 2012;28:556-64.

16. Sheeham A, Schmied V. The need for strategies that support women who are breastfeeding. In: Liamputtong P, editor Infant feeding practices: a cross-cultural perspective. London: Springer,2011:70-2.

17. Dykes F. Breastfeeding in hospital: mothers, midwives and the production line. London: Routledge; 2006.

18. Goulet C, Lampron A, Marcil I, Ross L. Attitudes and subjective norms of male and female adolescents toward breastfeeding. J Hum Lact. 2003;19:402-10.

19. Shehu M. Hassan Shehu. Knowledge, Attitude and Intending Practice on Breastfeeding Among Clinical Medical Students in Bingham University Teaching Hospital, Jos. European Journal of Preventive Medicine. 2019;7(2):50-6. doi:10.11648/j.ejpm.20190702.13.

20. Ahmed A, El Guindy SR. Breastfeeding knowledge and attitudes among Egyptian Baccalaureate students. Int Nurs Rev. 2011;58:372-78.

21. Ojofeitimi EO, Owolabi OO, Eni-Olorunda, Adesina OF, Esimai OA. Promotion of Exclusive Breastfeeding (EBF): The need to focus on the Adolescents. Nutr Health. 2001;15(1):55-62.

22. Kang NM, Song Y, Im EO. Korean university students' knowledge and attitudes toward breastfeeding: a questionnaire survey. Int J Nurs Stud. 2005;42:863-70.

23. Padmanabhan $\mathrm{R}$, Thulasingam M, Chinnakalai P. Female College Students knowledge, attitude and future intention towards breastfeeding: Implications for advocacy. Journal of Clinical and Diagnostic Research. 2016 Nov, Vol-10(11):LC11-4.

24. Anjum Q, Ashfaq T, Siddiqui H. Knowledge regarding breastfeeding practices among medical students of Ziauddin University Karachi. J Pak Med Assoc. 2007;57:480-3.

25. Hamade H, Naja F, Keyrouz S, Hwalla N, Karam J, Al-Rustom L, Nasreddine L. Breastfeeding knowledge, attitude, perceived behavior and intention of female undergraduate university students in the Middle East: The case of Lebanon and Syria. Food Nutr Bull. 2014;35(2):17990.

26. Osman H, El Zein L, Wick L. Cultural beliefs that may discourage breastfeeding among Lebanese women: a qualitative analysis. Int Breastfeed J. 2009;4(12):1-6.

27. Nabulsi M. Why are breastfeeding rates low in Lebanon? A qualitative study. BMC Pediat. 2011;11:75:1-6.

28. Acker M. Breast is best... but not everywhere: ambivalent sexism and attitudes toward private and public breastfeeding. Sex Roles. 2009;61:476-90.

29. Hannan A, Li RW, Benton-Davis S, Grummer-Strawn L. Regional variation in public opinion about breastfeeding in the United States. J Hum Lact. 2005;21:284-8.

30. Nelson AM. Adolescent attitudes, beliefs, and concerns regarding breastfeeding. Nursing. 2009;34:249-55.

31. Spear HJ. College students' experiences and attitudes regarding middle and high school-based breastfeeding education. J School Nurs. 2007;23:276-82.

32. Hamade H, Chaaya M, Saliba M, Chaaban R, Osman H. Determinants of exclusive breastfeeding in an urban population of primiparas in Lebanon: a cross-sectional study. BMC Public Health. 2013;13:702, 1-10.

33. Al-Akour NA, Khassawneh MY, Khader YS, Ababneh AA, Haddad AM. Factors affecting intention to breastfeed among Syrian and Jordanian mothers: a comparative cross-sectional study. Int Breastfeed J. 2010;5(6):1-8.

34. Montano DE, Kasprzyk D. Theory of reasoned action, theory of planned behaviour, and the integrated behavioural model. In: Glanz K, Rimer BK, Viswanath K, editors. Health behavior and health education: theory, research, and practice. San Francisco: Jossey-Bass; 2008. pp. 67-9.

35. Ahluwalia IB, Morrow B, Hsia J. Why do women stop breastfeeding? Findings from the pregnancy risk assessment and monitoring system. Pediatrics. 2005;116:1408-12.

36. Chezem J, Friesen C, Boettcher J. Breastfeeding knowledge, breastfeeding confidence, and infant feeding plans: Effects on actual feeding practices. J Obstet Gynecol Neonatal Nurs. 2003;32:40-7.

37. Li R, Fridinger F, Grummer-Strawn L. Public perceptions on breastfeeding constraints. J Hum Lact. 2002;18:227-35.

38. Bella H. Are Saudi female college students prepared for successful breastfeeding? [5] J R Soc Health. 1997;117(6):387-92.

39. James DC, Dobson B, American Dietetic Association. Position of the American Dietetic Association: promoting and supporting breastfeeding. J Am Diet Assoc. 2005;105:810-8.

40. Pérez-Rodrigo C, Klepp KI, Yngve A, Sjöström M, Stockley L, Aranceta J. The school setting: an opportunity for the implementation of dietary guidelines. Public Health Nutr. 2001;4:717-24. https://doi.org/10.1079/phn2001162. 
41. Friel JK, Hudson NI, Banoub S, Ross A. Th e effect of a promotion campaign on attitudes of adolescent females towards breastfeeding. Can J Public Health. 1989;80:195-9.

42. University of New South Wales Press

Sydney

Bartlett A. Breastwork. Sydney: University of New South Wales Press, 2005.

43. Wolfberg AJ, Michels KB, Shields W, O'Campo P, Bronner Y, Bienstock J. Dads as breastfeeding advocates: results from a randomized controlled trial of an educational intervention. Am J Obstet Gynecol. 2004;191:708-12.

\section{Figures}

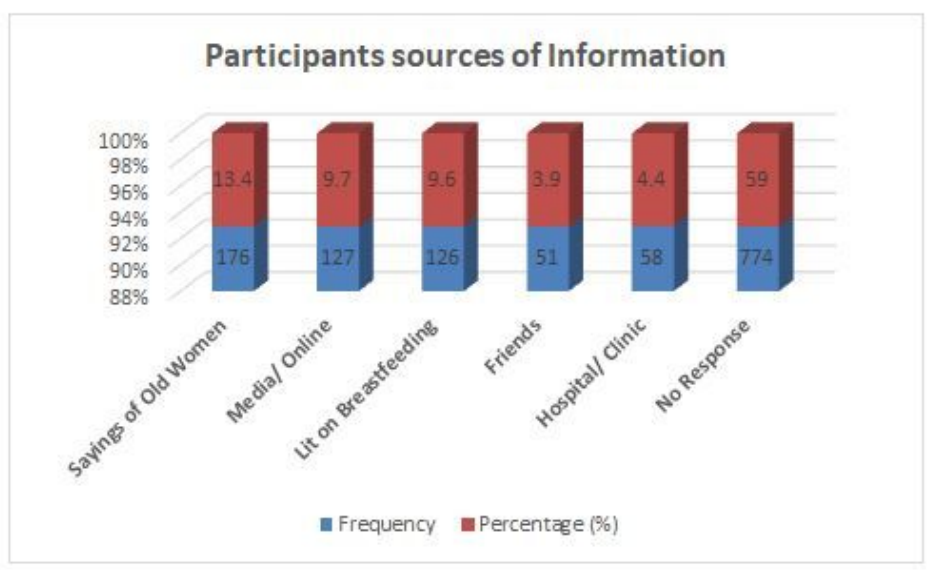

Figure 1

Participants sources of information about Breast milk and breastfeeding

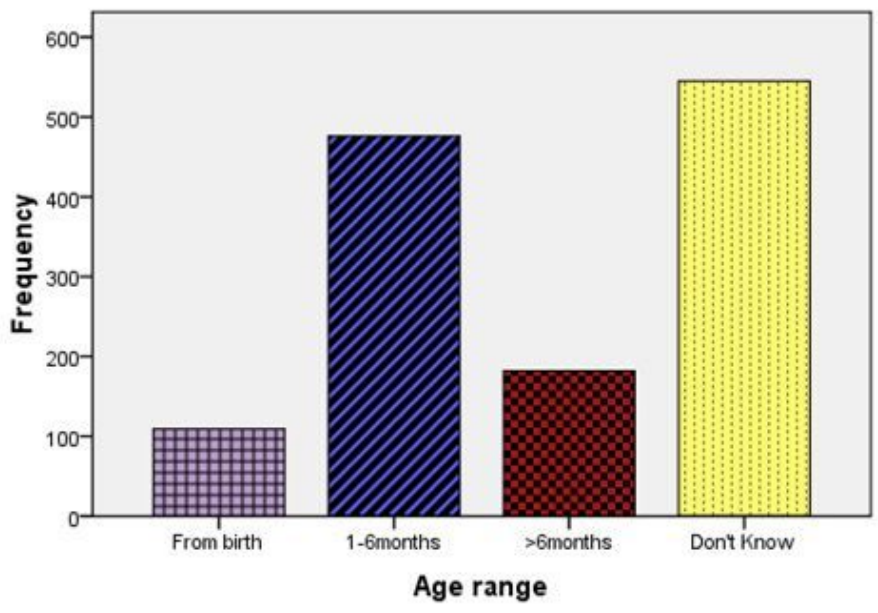

Figure 2

Age at introduction of water to infants on exclusive breastfeeding 


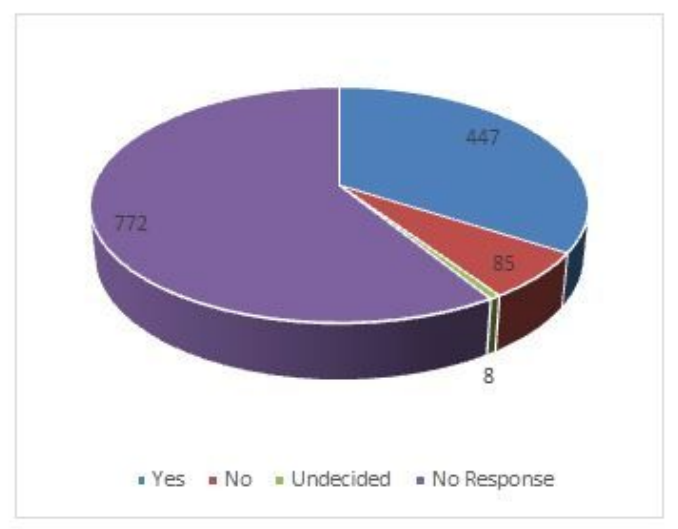

Figure 3

Intention to Breastfeed their future baby

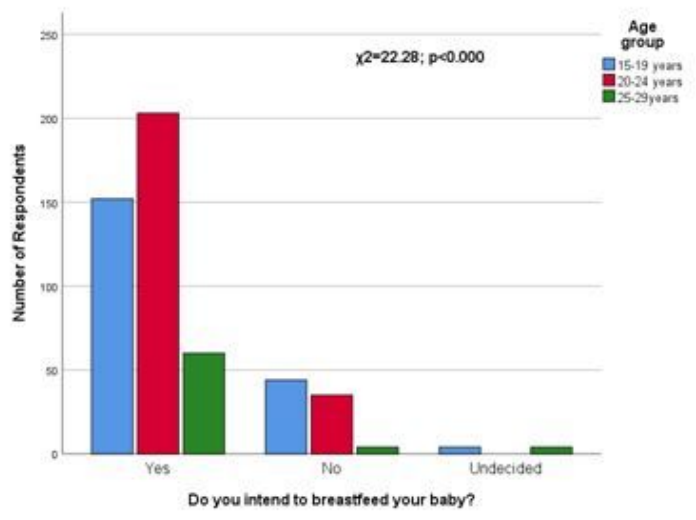

Figure 4

Intention to Breastfeed among Study participants 